\title{
"Sweet Chemistry": a Green Way for Obtaining Selenium Nanoparticles Active against Cancer Cells
}

\section{Adriana P. Vieira, ${ }^{*, a}$ Erika M. Stein, ${ }^{b}$ Daniel X. Andreguetti, ${ }^{c}$ Gerardo Cebrián-Torrejón, ${ }^{a, c}$ Antonio Doménech-Carbó, ${ }^{c}$ Pio Colepicolo ${ }^{d}$ and Ana Maria D. C. Ferreira ${ }^{a}$}

\author{
${ }^{a}$ Departamento de Química Fundamental, Instituto de Química and \\ ${ }^{b}$ Departamento de Análises Clínicas e Toxicológicas, Faculdade de Ciências Farmacêuticas, \\ Universidade de São Paulo, Av. Prof. Lineu Prestes, 03178-200 São Paulo-SP, Brazil
}

cDepartament de Química Analítica, Facultat de Química, Universitat de València, Dr. Moliner 50, 46100 Burjassot, Valencia, Spain

${ }^{d}$ Departamento de Bioquímica, Instituto de Química, Universidade de São Paulo, Av. Prof. Lineu Prestes, 748, 05508-000 São Paulo-SP, Brazil

\begin{abstract}
We present an environment friendly synthesis of selenium nanoparticles and the study of their cytotoxic activity against uterine sarcoma cancer and fibroblasts cells. Amorphous selenium (a-SeNPs) and trigonal selenium (t-SeNPs) were synthesized using $D$-fructose as the reducing agent and characterized by high-resolution transmission electron microscopy (HRTEM), energydispersive X-ray spectroscopy (EDX), powder X-ray diffraction analysis (XRD), inductively coupled plasma optical emission spectrometry (ICP OES), dynamic light scattering (DLS) to obtain zeta potential values and cyclic voltammetry (CV). Particularly, a-SeNPs presented high toxicity toward the resistant cancer cell line MES-SA/Dx5 and its parental MES-SA line. However, they are not toxic against P4 fibroblast cells in comparative studies.
\end{abstract}

Keywords: selenium nanoparticles, $D$-fructose, cancer cells, green chemistry, cytotoxicity

\section{Introduction}

Selenium is an essential element of significant importance not only for humans, but also for other forms of life..$^{1,2}$ Inorganic seleno-compounds have strong antioxidant activity, as well as some pro-oxidant effects and, additionally, have great importance in nutrition and medicine. ${ }^{3,4}$ Epidemiological studies have already demonstrated the potential role of selenium in the prevention and treatment of cancer cells..$^{5-7}$ For example, selenium supplementation showed effective reduction in the incidence of cancer in the cervix, lung and liver. ${ }^{8}$ However, the biological activity of elemental selenium stands opposite to the selenium salts, since selenite and selenate showed inadequate cytotoxicity to normal cells. ${ }^{9-11}$ In this sense, nanoparticles of elemental selenium stand out as an alternative, due to its low toxicity and acceptable bioavailability. ${ }^{12-14}$

*e-mail: apiresvieira@gmail.com

Conflict of interest: the University of São Paulo has filed patent (BR102016012928-1) relating to selenium nanoparticles synthesis and applications under study in the A. M. D. C. F. and P. C. laboratories.
Nanoparticles have become increasingly prominent in the medical field in the last few decades, giving rise to a new area, nanomedicine. This new area is focused on developing nanomaterials with potential use in therapy and diagnosis of diseases, ${ }^{15-17}$ including selenium nanoparticles (SeNPs). ${ }^{18}$

Generally, the methods for obtaining SeNPs involve multiple steps, high temperatures and pressures; require expensive equipment and reagents, as well as being harmful to the environment. ${ }^{19-27}$ In this sense, it is necessary to develop simple routes that focus on green chemistry and no harm to the environment. Research has demonstrated the use of microorganisms such as bacteria, to reduce selenite. This method is considered a clean synthesis, but the cost and time of preparation of microbiological cultures is a disadvantage. ${ }^{28-30}$

The use of plant extracts and biopolymers such as chitosan, konjac glucomannan, gum acacia, carboxymethyl cellulose, sodium alginate and glutathione, as reducing agents of selenite has also been an alternative, since they are highly biodegradable and non-toxic materials. However, in each of these works there are several processing steps 
until the attainment of SeNPs..$^{31-33}$

In this paper, we propose a method of synthesis of SeNPs that meet the principles of green chemistry, through a synthesis that requires little reaction time, non-toxic solvents, mild temperatures and uses a reducing agent that is biodegradable, cheap and not harmful to the environment. The use of sugars as reducing agents to obtain such nanoparticles has been investigated. ${ }^{34-36}$ Glucose and fructose have aldehyde and hydroxyl groups at carbon 1 , respectively, which make them effective reducing agents. Researchers used glucose as a reducing agent and stabilizer of SeNPs; however, in their synthesis they used high temperatures and toxic solvents. ${ }^{35,36}$

Therefore, we chose $D$-fructose because it is a plentiful and cheap reducing sugar to act as a reducing agent for sodium selenite in clean and quick synthesis of SeNPs using water as solvent and at low temperature. The obtained nanoparticles were tested for cytotoxicity against normal cells (fibroblasts-P4) and uterine sarcomas cells (MES-SA and MES-SA/Dx5) in order to assess their potential use as an anticancer drug.

\section{Experimental}

\section{Preparation and characterization of selenium nanoparticles (SeNPs)}

The SeNPs were prepared by the following procedure: an aqueous solution $\left(1.0 \mathrm{mmol} \mathrm{L} \mathrm{L}^{-1}, 5 \mathrm{~mL}\right)$ of sodium selenite (Merck, Darmstadt, Germany) was slowly dripped in $10.0 \mathrm{~mL}$ of $1.0 \mathrm{mmol} \mathrm{L}^{-1}$ aqueous solution of $D$-fructose (Merck, Darmstadt, Germany). The reaction mixture was then stirred, under heat at $45{ }^{\circ} \mathrm{C}$ for $15 \mathrm{~min}$, when red staining nanoparticles were obtained (amorphous selenium, a-SeNPs), turning black after 20 min (trigonal selenium, t-SeNPs). Each solution containing selenium nanoparticles (a-SeNPs and t-SeNPs) was centrifuged at $13,000 \mathrm{rpm}$ for 10 minutes. The pellets obtained were then re-suspended in deionized water and centrifuged again under the same conditions. This process was repeated three times for the removal of organic impurities present in SeNPs.

The nanoparticles free of organic impurities were quantified regarding selenium using an optical emission spectrometer with inductively coupled plasma (ICP OES) of Spectro, model Arcos. The energy-dispersive X-ray spectroscopy (EDX) analyses were obtained on a Shimadzu, EDX-720 model. The corresponding powder X-ray diffraction analysis (XRD) pattern was collected using a diffractometer with $\mathrm{Cu} \mathrm{K} \alpha$ operating at $30 \mathrm{kV}, 15 \mathrm{~mA}$, $5^{\circ}$ to $2 \theta=90^{\circ}$, Rigaku, Miniflex model. The morphology and particle size were analyzed using a high-resolution transmission electron microscope (HRTEM) JEOL, JEM 2100 model. The preparation of the samples consisted in dispersing the nanoparticles in a coated copper grid with a thin film of carbon, after careful drying with a filter paper. The grid was observed by HRTEM, using an accelerating potential of $200 \mathrm{kV}$. Measurements of the zeta potential were carried out by dynamic light scattering (DLS) using a Malvern, Zetasizer Nano model. Electrochemical experiments were performed at $298 \pm 1 \mathrm{~K}$ using potentiostat CH instruments, $\mathrm{CH}$ 660I model. A graphite bar (1 mm diameter) was used as working electrode, a platinum wire as auxiliary electrode and an $\mathrm{Ag} / \mathrm{AgCl}\left(3 \mathrm{~mol} \mathrm{~L}^{-1} \mathrm{NaCl}\right)$ reference electrode completing the three-electrode arrangement. Voltammetry of microparticles experiments were performed at microparticulate films of amorphous selenium (a-SeNPs) and trigonal selenium ( $t$-SeNPs) prepared by abrasively transferring a few nanogram sample onto the surface of the graphite electrode. Aqueous $0.25 \mathrm{~mol} \mathrm{~L}^{-1} \mathrm{HAc} / \mathrm{NaAc}$ buffer solution at $\mathrm{pH} 4.75$ was used as the electrolyte.

\section{Cytotoxicity assays}

For the in vitro studies of the cytotoxicity, the following cells were used: human foreskin fibroblasts isolated from tissue (cell P4), drug-sensitive human sarcoma cell line MES-SA and its multidrug-resistant (MDR) counterpart MES-SA/Dx5, as a model system for modulators' anti-MDR potency evaluation. $\mathrm{P} 4$ cells were maintained on Dulbecco's modified eagle medium (DMEM) containing $10 \%$ fetal calf serum, amphotericin B $\left(0.63 \mu \mathrm{g} \mathrm{mL}^{-1}\right)$ and penicillin $\left(100 \mathrm{IU} \mathrm{mL}^{-1}\right)$, streptomycin $\left(100 \mu \mathrm{g} \mathrm{mL}^{-1}\right)$, at $37{ }^{\circ} \mathrm{C}$, in a humidity-controlled incubator at $90 \%$ relative humidity and $5 \% \mathrm{CO}_{2}$. MES-SA cells were maintained on McCoy's 5A culture medium supplemented as above, and the MDR cell line MES-SA/Dx5 was cultivated at the same medium with doxorubicin $0.5 \mu \mathrm{M}$, at $37{ }^{\circ} \mathrm{C}$, in a humidity-controlled incubator at $90 \%$ relative humidity and $5 \% \mathrm{CO}_{2}$. After a few passages, cells were incubated at a concentration of $10 \times 10^{3}$ cell per wall for drug exposition of $24 \mathrm{~h}$ in 96-wall plates, with a final volume of $100 \mu \mathrm{L}$. After a period of 24 or $48 \mathrm{~h}$, the cells were treated with concentrations 10 to $140 \mu \mathrm{M}$ of the a-SeNPs. The mortality cell assay was performed in duplicate, $24 \mathrm{~h}$ after starting exposure to drugs. The tetrazolium reduction assay (MTT) was performed in triplicate, according to the literature method. ${ }^{37}$ Briefly, the medium was removed from the plates, the attached cells were washed with phosphate buffer solution and $200 \mu \mathrm{L}$ of the MTT solution were added to replaced culture medium, at a concentration of $500 \mu \mathrm{g} \mathrm{mL} L^{-1}$. After an incubation period of $4 \mathrm{~h}$ at $37^{\circ} \mathrm{C}$, the MTT solution 
was removed, and the formazan crystals were solubilized in $100 \mu \mathrm{L}$ of DMSO. Absorbance of this formazan solution was measured with a Tecan Infinite M200 microplate reader (Männendorf, Switzerland), at a wavelength of $570 \mathrm{~nm}$. The cell viability rate was estimated from the number of intact cells relative to the total number of cells in the corresponding control, and it was expressed as the percentage of viable cells. The $\mathrm{IC}_{50}$ was described as the concentration necessary to reach the mortality of $50 \%$ of cells.

\section{Results and Discussion}

Reduction of sodium selenite was clearly confirmed through observation with naked eye. A color change occurred, from colorless to a red solution within $15 \mathrm{~min}$ of reaction (Figure 1). This red coloring, which is characteristic of monoclinic colloidal selenium nanoparticles (a-SeNPs), is due to surface plasmon excitation. ${ }^{38}$ Within 20 min of reaction, black coloring nanoparticles were obtained, which is characteristic of selenium nanoparticles in the trigonal phase (t-SeNPs). ${ }^{39}$

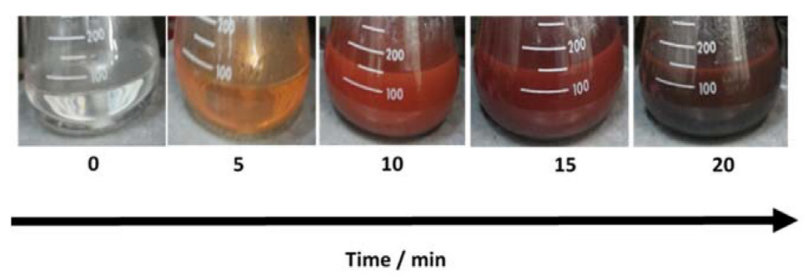

Figure 1. Selenium nanoparticles formation: reaction as a function of time.

Figure 2a shows the XRD pattern of the sample obtained after $15 \mathrm{~min}$ of reaction, which has no sharp peak, confirming that the selenium nanoparticles synthesized under these conditions were in the amorphous phase (a-SeNPs). ${ }^{24}$ In the sample obtained after 20 min of reaction, peaks were observed (Figure 2b) at $2 \theta=23,29,41,43,45$, $51,55,61$ and $65^{\circ}$, corresponding to the crystalline planes $100,101,110,102,111,201,112,103$. All the peaks could be indexed to the trigonal phase of selenium nanoparticles (t-SeNPs). ${ }^{40}$ The lattice constants of $\mathrm{a}=4.3662 \AA$ and $\mathrm{c}=4.9521 \AA$ are consistent with the standard values for bulk Se with $\mathrm{a}=4.3662 \AA$ and $\mathrm{c}=4.9536 \AA$, according to JCPDS file No. 73-0465.

The HRTEM images corroborate the X-ray diffraction data, which show selenium nanoparticles obtained at $15 \mathrm{~min}$ of the reaction having spherical shape without aggregation of particles (Figures 3A and 3B). This is characteristic of the amorphous phase $\mathrm{e}^{30,31}$ (a-SeNPs) with the size ranging between $80-100 \mathrm{~nm}$, and average particle size at $80 \mathrm{~nm}$ (Figures $3 \mathrm{~A}$ and 3B). Figures $3 \mathrm{C}$ and 3D show that the shape of the nanoparticle depends on the reaction time.

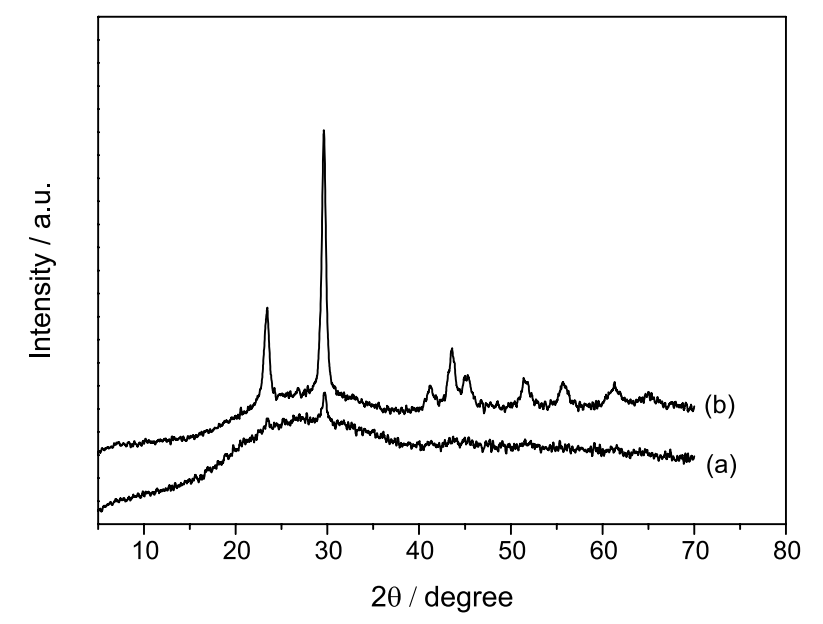

Figure 2. XRD pattern of selenium nanoparticles prepared using $D$-fructose after $15 \mathrm{~min}$ reaction, a-SeNPs (a), and after 20 min reaction, t-SeNPs (b).

After 20 min of reaction, the amorphous Se spheres (a-SeNPs) tend to form aggregates. Then, the particles start crystallization presenting rod-like shape, which can be characteristic of t-SeNPs. ${ }^{30}$ This behavior was observed in other studies in which conventional methods of synthesis of selenium nanoparticles were used. ${ }^{24}$

The EDX analysis shows qualitatively and quantitatively the elements that may be involved in the formation of nanoparticles. Figure 4 shows the high purity of the selenium nanoparticles synthesized. The two peaks at 11.2 and $12.1 \mathrm{keV}$, are related to the presence of selenium. ${ }^{31}$

The nanoparticle stability is very important for a number of applications, and can be determined through the zeta potential. For a-SeNPs synthesized in this work, the zeta potential was found to be $-27.8 \pm 0.5 \mathrm{mV}$. This result indicates the high stability of monodisperse nanoparticles..$^{32,41}$ To the nanoparticles obtained after 20 min of reaction, t-SeNPs, the measured zeta potential was $-18.2 \pm 0.9 \mathrm{mV}$. This confirms the results obtained by the HRTEM image, which showed polydisperse particle aggregation, and indicates the poor stability of them.

Figure 5 compares the voltammetric responses of nanoparticulate deposits of a-SeNPs and t-SeNPs attached to graphite immersed into $0.25 \mathrm{~mol} \mathrm{~L}^{-1} \mathrm{HAc} / \mathrm{NaAc}$ aqueous buffer. Using square wave voltammetry as a detection mode, both oxidation and reduction signals can be seen in positive-going scans. Upon scanning the potential from $-0.85 \mathrm{~V}$ vs. $\mathrm{Ag} / \mathrm{AgCl}$ in the positive direction, the a-SeNPs displayed voltammetric signals at $-0.80,-0.20$ and a shoulder at $+0.95 \mathrm{~V}$. Remarkably, the t-SeNPs produced weaker shoulders at $-0.80,-0.20$ and $+0.10 \mathrm{~V}$ intercalated between a sharp peak at -0.45 and a broad wave at $+0.95 \mathrm{~V}$. These responses can be interpreted in terms of the occurrence of two different electrochemical 

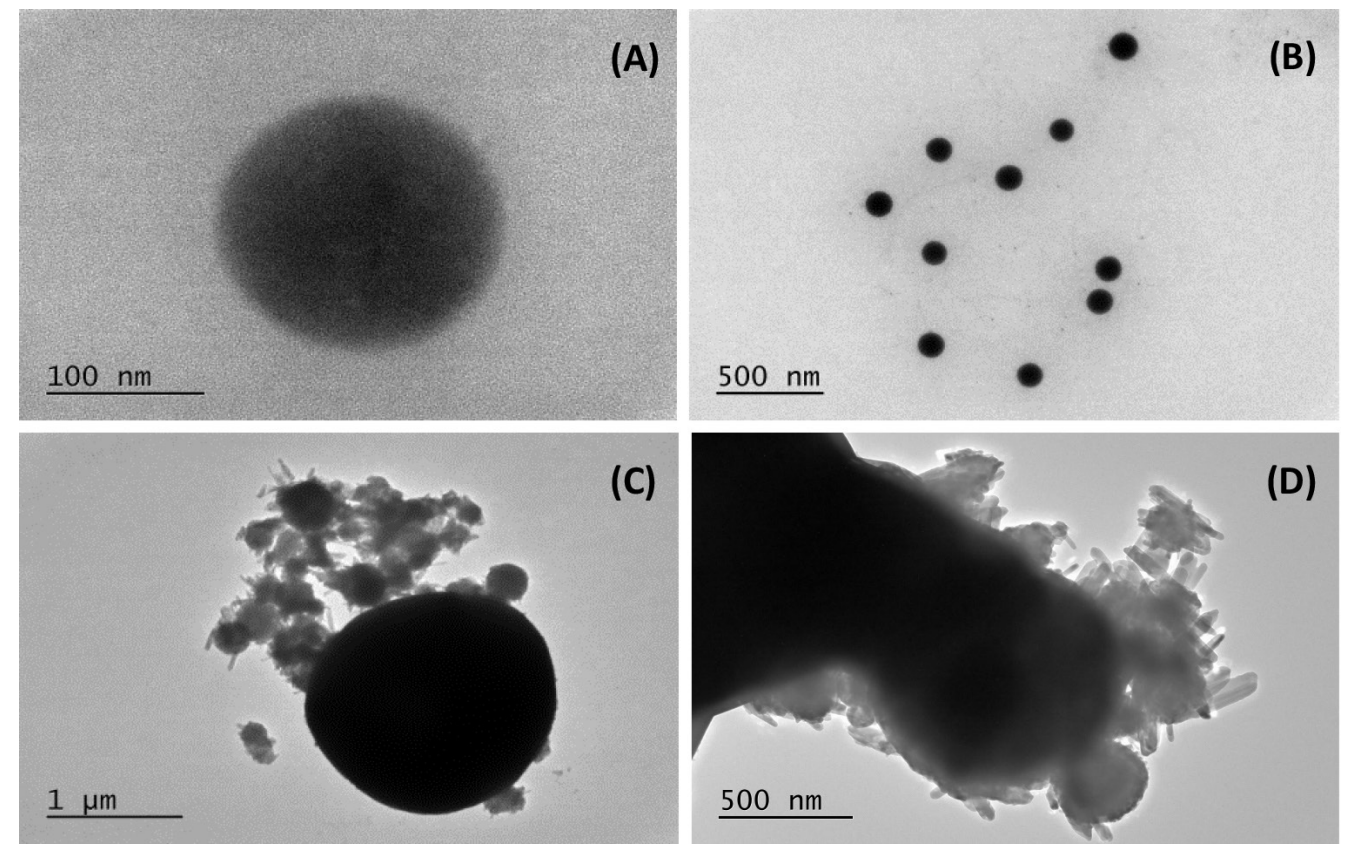

Figure 3. HRTEM images of the transformation process of a-SeNPs nanospheres to t-SeNPs nanorods. (A) a-SeNPs individual nanosphere ; (B) uniform nanospheres of a-SeNPs; (C) aggregation and beginning of the transformation into t-SeNPs nanorods; (D) nanorods aggregates of t-SeNPs.

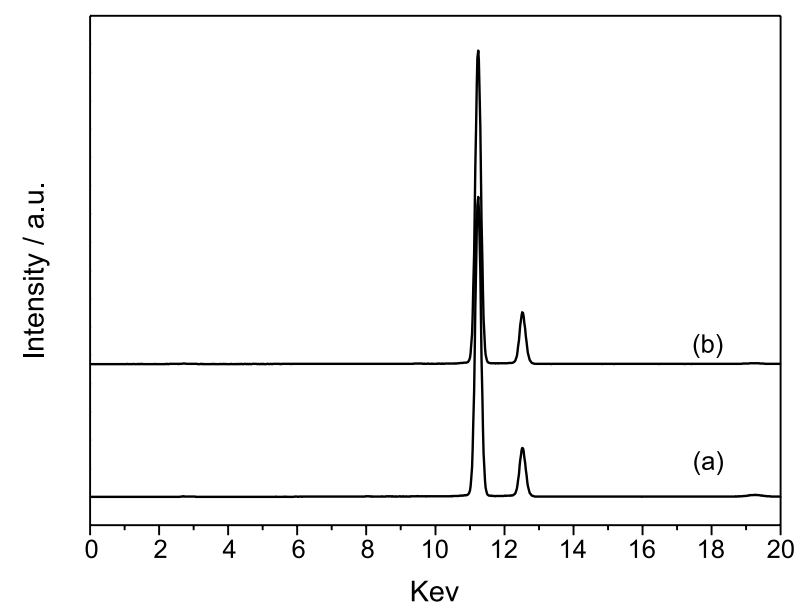

Figure 4. EDX spectrum of a-SeNPs after 15 min reaction (a) and t-SeNPs after $20 \mathrm{~min}$ (b).

pathways. First of all, the common signal at $-0.80 \mathrm{~V}$ can be attributed to the reduction of Se to $\mathrm{H}_{2} \mathrm{Se}^{42}$ The oxidation of SeNPs, however, occurs differently for a- and t-SeNPs. In this second case, a sharp signal at $-0.45 \mathrm{~V}$ appears, corresponding to a typical stripping process ${ }^{43}$ due to the oxidation of selenium( 0 ) to selenium(II) species in solution. In contrast, the oxidation of a-SeNPs gives rise to a broad peak at $-0.20 \mathrm{~V}$ which can be attributed to the selenium(0) oxidation to NP-associated selenium(II). Such processes would precede the oxidation of selenium( $(0)$ and selenium(II) to selenium(IV), producing the oxidation signals at more positive potentials. ${ }^{44-46}$

These features can be rationalized assuming that selenium $(0)$ oxidation is quite sensitive to the crystalline

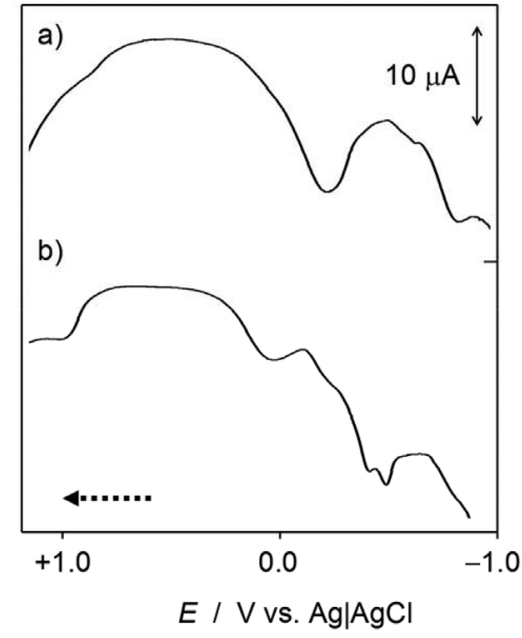

Figure 5. Square wave voltammograms of nanoparticulate deposits of: (a) a-SeNPs and (b) t-SeNPs attached to graphite electrode, immersed into $0.25 \mathrm{~mol} \mathrm{~L}^{-1} \mathrm{HAc} / \mathrm{NaAc}$ buffer solution. Potential scan initiated at $-0.85 \mathrm{~V}$, in the positive direction. Potential step increment, $4 \mathrm{mV}$; square wave amplitude, $25 \mathrm{mV}$; frequency, $5 \mathrm{~Hz}$.

structure, as observed in the electrochemistry of, for instance, gold NPs. ${ }^{47,48}$ In the case of t-SeNPs, which have a rigid structural arrangement, the electrochemical oxidation is dominated by the process at $-0.45 \mathrm{~V}$ which yields mainly selenium(II) in solution. In contrast, the oxidation of a-SeNPs, where a more flexible coordinative environment for Se atoms exists, can result in a smoother oxidation (process at $-0.20 \mathrm{~V}$ ), producing selenium(II) species associated to the NP. In summary, the availability of electroactive sites cycling between the selenium(0) and selenium(II) states is the key difference between the a- and t-SeNPs. 


\section{Cytotoxicity data}

The colloidal selenium nanoparticles a-SeNPs, obtained after $15 \mathrm{~min}$ of reaction, were evaluated for potential antitumor cytotoxicity against the cell line of uterine cancer MES-SA/Dx5 (doxorubicin-resistant mutants, with P-gp overexpression), and its parental cell line MES-SA. In addition, they were also tested for fibroblasts cell line P4. As shown in Figure 6A, the a-SeNPs were not able to affect P4 cells significantly, since the observed cell viability was 98 and $85 \%$ after 24 and 48 h of incubation, respectively, at a concentration of $140 \mu \mathrm{M}$. This data indicates that the selenium nanoparticles are non-toxic for this type of human healthy cell. In contrast, the results for cancer cells, shown in Figures $6 \mathrm{~B}$ and $6 \mathrm{C}$ indicate that a-SeNPs present high toxicity with respect to the sarcoma cells. Cell viability decreases gradually with increasing concentration, and the activity is higher with increasing incubation time, for both cancer cell lines studied. $\mathrm{IC}_{50}$ values were very similar, $110.01 \pm 0.23$ and $80.12 \pm 0.41 \mu \mathrm{M}$, at 24 and $48 \mathrm{~h}$, respectively, for

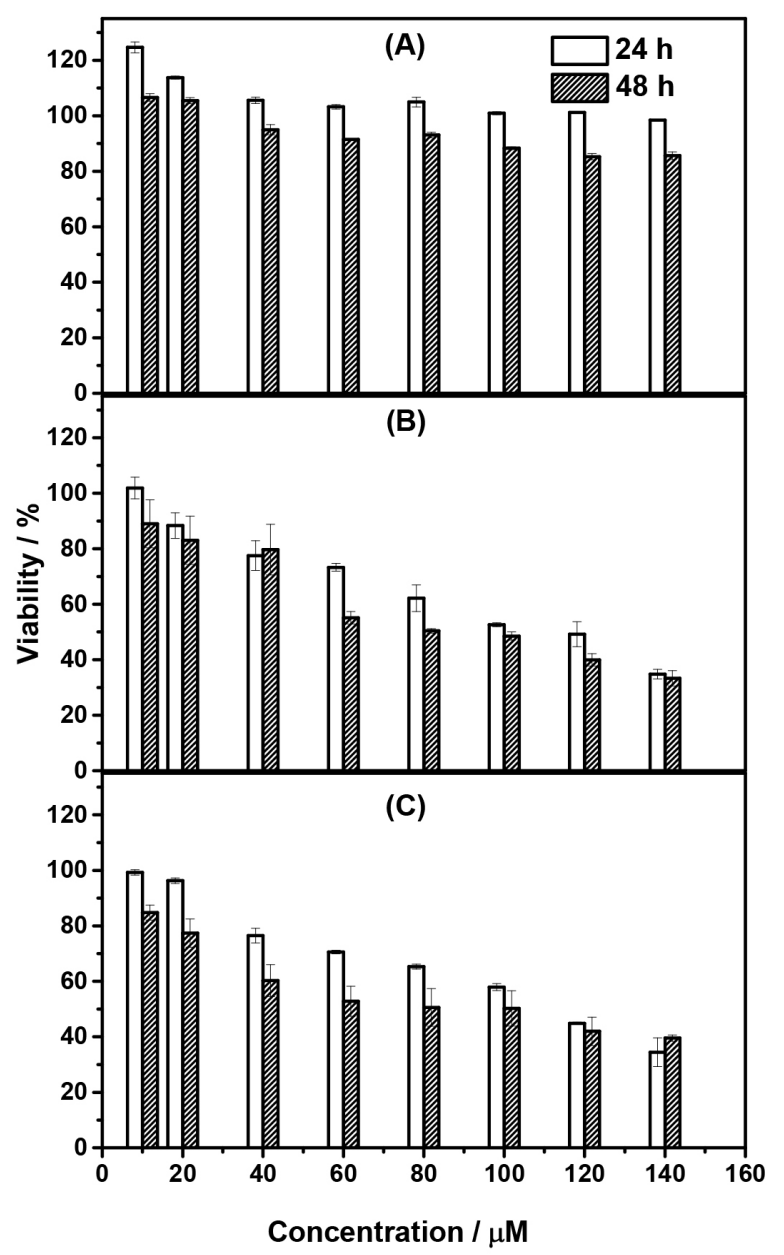

Figure 6. Effect of a-SeNPs synthesized by reduction with $D$-fructose on the viability of (A) P4 cell lines; (B) MES-SA cells and (C) MES-SA/Dx5.
MES-SA and $110.19 \pm 0.12$ and $81.38 \pm 0.34 \mu \mathrm{M}$, at 24 and $48 \mathrm{~h}$, respectively, for MES-SA/Dx5. The MES-SA/Dx5 cells usually overexpress P-glycoproteins, which act as a drug efflux pump. Consequently, these cells require higher doses of cytotoxic agent in comparison to the non-resistant cells. ${ }^{49}$ Therefore, these results show that the nanoparticles are equally toxic toward both the MDR (MES-SA/Dx5) and the non-resistant (MES-SA) cancer cell lines. This makes a-SeNPs a promising candidate to pharmaceutical agents to overcome resistance in cancer cells.

A previous study in the literature ${ }^{50}$ showed that SeNPs coupled with adenosine triphosphate (ATP, 2.5-40 $\mu \mathrm{M}$ ) inhibit the cellular viability of hepatocellular cancer strain (HepG2), but do not exhibit toxicity to normal human kidney cells (HK-2). In a more recent paper, ${ }^{51}$ SeNPs were applied to HCT- 8 cells (ileocecal adenocarcinoma), indicating that after $48 \mathrm{~h}$ of treatment only $27 \%$ of the cells survive, when $200 \mu \mathrm{M}$ of SeNPs were used. Associated with irinotecan, SeNPs are able to inhibit the growth of this strain more efficiently compared to each irinotecan or SeNPs alone. Concerning normal cells (IEC6), the SeNPs were less toxic than irinotecan. ${ }^{51}$ Breast cancer cells (MCF-7 and MDA-MB-231) were also treated with SeNPs, and an $\mathrm{IC}_{50}$ value of $25 \mu \mathrm{g} \mathrm{mL}^{-1}$ was obtained versus the MCF-7 line. For the MDA-MB-231 cell line, the inhibition was only $33 \%$ when the same maximum concentration was used, suggesting that SeNPs possess greater selectivity for early stage breast cancer compared to the metastatic breast cancer cells (MDA-MB-231). ${ }^{33}$ SeNPs (10-40 $\left.\mu \mathrm{M}\right)$ synthesized using vitamin $\mathrm{C}$ also showed activity against the lines MDA-MB-231 and HeLa (human cervical carcinoma). The viability of MDA-MB-231 cells was decreased to $60 \%$ and of HeLa cells was reduced between 73 and $50 \%$, in the presence of Se-NPs. ${ }^{52}$ This is the first time that MES-SA and MES-SA/Dx 5 cell lines are tested in studies with selenium nanoparticles. The obtained results confirmed the overall trends reported in the literature. As here discussed, SeNPs have great potential in the treatment against cancer cells, due mainly to its low toxicity toward normal cells. In addition, these results are quite attractive in terms of selectivity, since SeNPs were capable of inducing the mortality of MDR tumor cells (MES-SA/Dox5), which overexpress the glycoprotein P-gp responsible for the efflux effect in tumor cells, at the same intensity as compared with the standard MES-SA cells (Table 1).

\section{Conclusions}

In this study, we described a "green" synthesis of SeNPs using $D$-fructose as a reducing agent, by a simple, economic and fast process. This is the first report of 
Table 1. Cytotoxicity of SeNPs against different cell lines

\begin{tabular}{lcccc}
\hline Cell line & $\begin{array}{c}\text { Incubation } \\
\text { time / } h\end{array}$ & $\mathrm{IC}_{50} / \mu \mathrm{M}$ & $\begin{array}{c}\text { Size average } \\
\text { diameter } / \mathrm{nm}\end{array}$ & Reference \\
\hline Normal & & & & \\
\hline HK-2 & 24 & $>40$ & 1000 & 50 \\
IEC6 & 48 & non-toxic & 20 & 51 \\
P4 & 24 and 48 & $>140$ & 80 & this work \\
\hline Cancer & & & & \\
\hline HepG2 & 24 & ca. 7.5 & 1000 & 50 \\
HCT-8 & 48 & ca. 50 & 20 & 51 \\
MCF-7 & 24 & 310 & 150 & 33 \\
MDA-MB-231 & 24 & 1265 & 150 & 33 \\
MDA-MB-231 & 24 & $>506$ & 133 & 52 \\
HeLa & 24 & $>506$ & 133 & 52 \\
MES-SA & 48 & 80 & 80 & this work \\
MES-SA/Dx5 & 48 & 80 & 80 & this work \\
\hline
\end{tabular}

$\mathrm{IC}_{50}$ : the concentration necessary to reach the mortality of $50 \%$ of cells.

synthesis of Se nanoparticles where $D$-fructose has been used. Characterization techniques used indicate that at 15 min of reaction colloidal nanoparticles (a-SeNPs) were obtained, and in 20 minutes the process of transformation of a-SeNPs to t-SeNPs starts. HRTEM showed that a-SeNPs were obtained with $80 \mathrm{~nm}$ average particle size. Their purity was confirmed by EDX. Further, the a-SeNPs are highly stable with determined zeta potential of $(-27.8 \pm 0.5 \mathrm{mV})$. These data indicate that the electron transfer ability of the a-SeNPs and t-SeNPs nanoparticles is clearly different. Those results are in agreement with the observed cytotoxic data, since selenium nanoparticles induce pronounced death of cancer cells (MES-SA/Dx5 and MES-SA cells), but did not show noticeable antiproliferative effects on fibroblasts cells (P4) growth. These results indicate that SeNPs can be good candidates as new therapeutic drugs for uterine cancer treatment.

\section{Acknowledgments}

Financial support from the Brazilian entities Coordenação de Aperfeiçoamento de Pessoal de Nível Superior (CAPES), São Paulo State Research Foundation (FAPESP), the Ministerio de Ciência e Innovación (MICIN) Project CTQ2014-53736-C3-2-P, which is also supported with European Regional Development Funds, is gratefully acknowledged.

\section{References}

1. Rayman, M. P.; Lancet 2000, 356, 233.
2. Comasseto, J. V.; J. Braz. Chem. Soc. 2010, 21, 2027.

3. Bao, Y. P.; Williamson, G.; Prog. Nat. Sci. 2000, 10, 321.

4. Shen, H. M.; Yang, C. F.; Ding, W. X.; Liu, J.; Ong, C. N.; Free Radical Biol. Med. 2001, 30, 9.

5. Sinha, R.; Ei-Bayoumy, K.; Curr. Cancer Drug Targets 2004, 4, 13.

6. Ellis, R. D.; Salt, E. D.; Curr. Opin. Plant Biol. 2003, 6, 273.

7. Tapiero, H.; Townsend, D. M.; Tew, K. D.; Biomed. Pharmacother. 2003, 57, 134.

8. Abdulah, R.; Miyazaki, K.; Nakazawa, M.; Koyama, H.; J. Trace Elem. Med. Biol. 2005, 19, 141.

9. Huang, B.; Zhang, J. S.; Hou, J. W.; Chen, C.; Free Radical Biol. Med. 2003, 35, 805.

10. Wang, H. L.; Zhang, J. S.; Yu, H. Q.; Free Radical Biol. Med. 2007, 42, 1524.

11. Tarze, A.; Dauplais, M.; Grigoras, I.; Lazard, M.; Ha-Duong, N. T.; Barbier, F.; Blanquet, S.; Plateau, P.; J. Biol. Chem. 2007, $282,8759$.

12. Zhang, J. S.; Wang, H. L.; Yan, X. X.; Zhang; L. D.; Life Sci. 2005, 76, 1099.

13. Peng, D.; Zhang, J.; Liu Q.; Taylor, E. W.; J. Inorg. Biochem. 2007, 101, 1457.

14. Zhou, X. X.; Wang, Y. B.; Gu, Q.; Li, W. F.; Aquaculture 2009, 291, 291.

15. Acharya, S.; Dilnawaz, F.; Sahoo, S. K.; Biomaterials 2009, $30,5737$.

16. Patra, H. K.; Banerjee, S.; Chaudhuri, U.; Lahiri, P.; Dasgupta, A. K.; Nanomedicine 2007, 3, 111.

17. Durán, N.; Marcato, P. D.; de Conti, R.; Alves, O. L.; Costa, F. T. M.; Brocchi, M.; J. Braz. Chem. Soc. 2010, 21, 949.

18. Wadhwani, S. A.; Shedbalkar, U. U.; Singh, R.; Chopade, B. A.; Appl. Microbiol. Biotechnol. 2016, 100, 2555.

19. Cao, X. B.; Xie, Y.; Li, L. Y.; Adv. Mater. 2003, 15, 1914.

20. Ma, Y. R.; Qi, L. M.; Shen, W.; Ma, J. M.; Langmuir 2005, 21, 6161.

21. Chen, Z. X.; Shen, Y. H.; Xie, A. J.; Zhu, J. M.; Wu, Z. F.; Huang, F. Z.; Cryst. Growth Des. 2009, 9, 1327.

22. Yang, L. B.; Shen, Y. H.; Xie A. J.; Liang, J. J.; Eur. J. Inorg. Chem. 2007, 4438.

23. Li, S. K.; Shen, Y. H.; Xie, A. J.; Yu, X. Y.; Zhang, X. Z.; Yang, L. B.; Li, C. H.; Nanotechnology 2007, 18, 405101.

24. Song, J. M.; Zhu, J. H.; Yu, S. H.; J. Phys. Chem. B 2006, 110, 23790.

25. Quintana, M.; Haro-Poniatowski, E.; Morales, J.; Batina, N.; Appl. Surf. Sci. 2002, 195, 175.

26. Wang, M. C. P.; Zhang, X.; Majidi, E.; Nedelec, K.; Gates, B. D.; ACS Nano 2010, 4, 2607.

27. Filippo, E.; Manno, D.; Serra, A.; Cryst. Growth Des. 2010, 10, 4890.

28. Avendaño, R.; Chaves, N.; Fuentes, P.; Sánchez, E.; Jiménez, J. I.; Chavarría, M.; Sci. Rep. 2016, 6, 37155. 
29. Wang, T.; Yang, L.; Zhang, B.; Liu, J.; Colloids Surf., B 2010, $80,94$.

30. Zhang, W.; Chen, Z.; Liu, H.; Zhang, L.; Gao, P.; Li, D.; Colloids Surf., B 2011, 88, 196.

31. Prasad, K. S.; Patel, H.; Patel, T.; Patel, K.; Selvaraj, K.; Colloids Surf., B 2013, 103, 261.

32. Zhang, S.-Y.; Zhang, J.; Wang, H.-Y.; Chena, H.-Y.; Mater. Lett. 2004, 58, 2590 .

33. Vekariya, K. K.; Kaur, J.; Tikoo, K.; Nanomedicine 2012, 8, 1125.

34. Nie, T.; Wu, H.; Wong, K.-H.; Chen, T.; J. Mater. Chem. B 2016, 4, 2351.

35. Chen, H.; Yoo, J.-B.; Liu, Y.; Zhao, G.; Electron. Mater. Lett. 2011, 7, 333.

36. Chen, H.; Shin, D.-W.; Nam, J.-G.; Kwon, K.-W.; Yoo, J.-B.; Mater. Res. Bull. 2010, 45, 699.

37. Denizot, F.; Lang, R.; J. Immunol. Methods 1986, 89, 271.

38. Lin, Z. H.; Wang, C. R. C.; Mater. Chem. Phys. 2005, 92, 591.

39. Zhang, J. S.; Wang, H. L.; Bao, Y. P.; Zhang, L.; Life Sci. 2004, $75,237$.

40. Gates, B.; Mayers, B.; Cattle, B.; Xia, Y.; Adv. Funct. Mater. 2002, 12, 219.

41. Kong, H.; Yang, J.; Zhang, Y.; Fang, Y.; Nishinaria, K.; Phillips, G. O.; Int. J. Biol. Macromol. 2014, 65, 155.
42. Santos, M. C.; Machado, S. A. S.; J. Electroanal. Chem. 2004, $567,203$.

43. Lovric, M. In Electroanalytical Methods; Scholz, F., ed.; Springer: Berlin, 2002.

44. Beni, V.; Collins, G.; Arrigan, D. W. M.; Anal. Chim. Acta 2011, 699, 127.

45. Fierro, S.; Watanabe, T.; Akai, K.; Yamanuki, M.; Einaga, Y.; Int. J. Electrochem. 2012, 758708.

46. Feng, Y.; Gu, M.; Electrochim. Acta 2013, 90, 416.

47. Chen, A.; Lipkowski, J.; J. Phys. Chem. B 1999, 103, 682.

48. Burke, L. D.; O’Mullane, A. P.; J. Solid State Electrochem. 2000, 4, 285.

49. Vieira, A. P.; Stein, E. M.; Andreguetti, D. X.; Colepicolo, P.; Ferreira, A. M. C.; J. Appl. Phycol. 2016, 28, 2615.

50. Zhang, Y.; Li, X.; Huang, Z.; Zheng, W.; Fan, C.; Chen, T.; Nanomedicine 2013, 9, 74.

51. Gao, F.; Yuan, Q.; Gao, L.; Cai, P.; Zhu, H.; Liu, R.; Wang, Y.; Wei, Y.; Huang, G.; Liang, J.; Gao, X.; Biomaterials 2014, 35, 8854.

52. Luo, H.; Wang, F.; Baia, Y.; Chen, T.; Zheng, W.; Colloids Surf., $B$ 2012, 94, 304.

Submitted: August 26, 2016

Published online: February 13, 2017 